\title{
Desarrollos en el campo de los refractarios destinados a la industria del cemento
}

\author{
D. OPITZ \\ Instituto de investigaciones de la industria del cemento, Düsseldorf \\ Zement-Kalk-Gips, n 7, julio 1970, págs. 297-302
}

La industria del cemento consume del 5 al $15 \%$ de los productos refractarios fabricados en Alemania. La figura 1 muestra las cifras de producción de la industria de refractarios alemana entre los años 1960 a 1968, según datos de los anuarios estadísticos (1). La producción se rige por el curso de los negocios del mayor comprador, la industria del hierro y del acero, que por término medio consumen el $65 \%$ de dicha producción. Del aumento medio del costo, indicado asimismo en la figura 1, en DM por tonelada de material refractario -en total, alrededor de un $37 \%$-, recae aproximadamente $28 \%$ en mayores costos de producción y aproximadamente $9 \%$ en un consumo creciente de ladrillos de mejor calidad. En la construcción de hornos se va cada vez más a ladrillos de

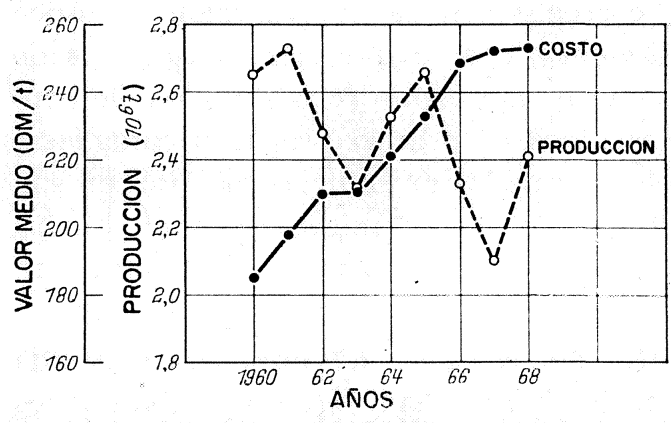

Fig. 1.-Producción y costo de los productos refractarios fabricados en la República Federal Alemana (datos del Negociado Federal de Estadística). mayor durabilidad o más definida a fin de evitar paradas imprevistas y planear con la mayor antelación posible las reparaciones del revestimiento.

En la industria del cemento se quiere poder producir hasta el próximo invierno con el revestimiento revisado, y en su caso reparado, en el invierno anterior. Si se ha elegido el ladrillo apropiado en la inspección invernal siguiente, o se encuentran tan desgastados que pueden desmontarse sin pérdida sustancial de material, o están en tan buen estado, que es seguro que puedan soportar otro viaje anual del horno. Sobre todo, en los grandes hornos rotatorios con una producción de clínker de más de 2.000 t/día, una parada imprevista de varios días origina, por la pérdida de producción, mayores gastos que una reparación preventiva.

Los costos de jornales de albañiles y de material refractario, que normalmente son del orden del $2 \%$ de los gastos de fabricación del cemento, no debieran valorarse por lo tanto más elevados que la seguridad del funcionamiento de la instalación. Por el mismo motivo se pasa hoy también a materiales más caros en zonas críticas del horno, como por ejemplo a ladrillos de carburo de silicio en la boca de salida del horno, siempre con la esperanza de lograr mayores tiempos de funcionamiento. 


\section{MASAS REFRACTARIAS}

Según la figura 2, dibujada de acuerdo con datos de Routschaka (2), la expedición de ladrillos refractarios ha disminuido un $24 \%$ entre 1965 y 1967 ; en cambio, la expedición de masas preparadas sólo un $13 \%$. Por consiguiente, aumenta la proporción de estas últimas. También la industria del cemento consume, desde hace años y con buen resultado, masas inyectadas y apisonadas para reparaciones y nuevos montajes de caperuzas de hornos, precalentadores y enfriadores.

En el tubo rotativo no se ha revestido hasta ahora monolíticamente más que la entrada del horno y las zonas de cadenas y cruces de los hornos largos. En las otras partes del tubo sólo se efectuaron, con resultados muy distintos, inyecciones para reparación de la mampostería fija. En diversas ocasiones se han intentado inyecciones de reparación con el horno caliente tanto en la zona de salida del horno y en la caperuza como en la zona de sinterización con el horno frío, pero, hoy día, estas medidas suponen soluciones provisionales a fin de retardar algunas semanas la reparación en frío. En suma, el empleo de masas en hornos de cemento no se ha impuesto en la medida deseada. Esto se debe seguramente a que aún no se ha podido reunir suficiente experiencia en la ejecución de elementos monolíticos.

Al montarse las masas interesa tanto el anclaje correcto con la construcción metálica como un secado esmerado y un calentamiento cuidadoso, con lo que ciertos agujeros de evaporación y algunas juntas previstas ayudan a evitar la posible rotura de los elementos constructivos al calentarse; al efectuarse reparaciones es preciso preparar cuidadosamente la base. La utilización de masas es ventajosa porque las existencias de almacén son más reducidas, no se depende de formatos y puede montarse un mismo tipo en varios lugares de la instalación. Además los elementos monolíticos tienen menos juntas y, en consecuencia, menos puntos débiles.

Por último, para la colocación de masas se necesita menos mano de obra especializada que para la mampostería. Caperuzas apisonadas en los hornos han logrado ya duraciones más largas que las construidas con ladrillos de la misma composición.

\section{LADRILLO EMPLEADO EN EL TUBO ROTATORIO}

La elección de los materiales para el revestimiento del tubo rotatorio sigue siendo problemática, ya que no es posible, basándose en los valores de ensayo de los fabricantes, encontrar el ladrillo idóneo para el lugar de utilización. En la tabla 1 se indican los grupos de ladrillos usuales para las distintas zonas de un horno rotatorio corto. De ella se deduce que, sobre todo en el anillo de descarga, se colocan tipos de ladrillos completamente distintos tanto química como físicamente.

En los últimos 20 años ha tenido lugar un cambio sustancial en los tipos de ladrillo para la zona de sinterización. Hasta la mitad de los años 50 se emplearon preferentemente ladrillos con alto contenido de alúmina y ladrillos de magnesita. Se pasó después a la- 
drillos de cromo-magnesita y ladrillos de magnesita; hoy en día se utilizan preferentemente ladrillos de dolomía. En el extranjero no se ha producido este desarrollo al unísono; se calcula, por ejemplo, que la mitad de las zonas de sinterización en los hornos de cemento de USA se hallan todavía actualmente revestidos con ladrillos de alto contenido en alúmina, y la otra mitad principalmente con ladrillos de magnesita y cromomagnesita.

Zonas críticas con posibilidad de averías imprevistas son, sobre todo, el anillo de descarga y la zona de transición. En el anillo de descarga los materiales que mejor resisten en condiciones difíciles son los ladrillos de $\mathrm{SiC}$ con aproximadamente $60 \%$ de contenido de $\mathrm{SiC}$. La duración de los ladrillos del anillo de descarga viene dada frecuentemente por la vida de la zona metálica de la salida del horno. Al cambiar este sector hay que quitar también roscas de ladrillo todavía intacto, siendo así que la larga duración de ladrillos de gran calidad no se aprovecha frecuentemente de ningún modo.

Ultimamente, los llamados ladrillos de cromo-magnesita de alta cocción "aglomerados directamente" han soportado las difíciles condiciones en la zona de transición. Estos ladrillos se fabrican de materias primas seleccionadas y se cuecen a $1.800^{\circ} \mathrm{C}$ en vez de a $1.650^{\circ} \mathrm{C}$, como se hace normalmente, a fin de aumentar su resistencia al fuego y hacerlos más resistentes contra infiltraciones y atmósferas de gases reductoras.

\section{PROPIEDADES DE LOS LADRILLOS REFRACTARIOS}

Dentro de los distintos grupos de ladrillos, por ejemplo ladrillos de magnesita o con alto contenido de alúmina, se ofrece una multitud de clases. Para tener una idea de la oferta, se confeccionó un catálogo de materiales refractarios para fábricas de cemento. Contiene los datos técnicos de 140 clases de ladrillos y 90 clases de masas, que ofrecen 16 fabricantes alemanes y 11 extranjeros y que casi todos han sido probados ya en fábricas de cemento alemanas.

Con arreglo a una propuesta de la P.R.E. (Federación Europea de Fabricantes de Productos Refractarios), se distingue entre productos compactos (porosidad total $<45$ $\%$ ), ladrillos refractarios ligeros y ladrillos aislantes. La siguiente subdivisión se hace de acuerdo con los principales componentes quimicos. La tabla 2 muestra esta clasificación y la gama de propiedades de los distintos grupos de ladrillos. A continuación se analizará hasta dónde existen relaciones entre los valores de las distintas propiedades.

La máxima temperatura posible a aplicar de masas y ladrillos conteniendo chamota y $\mathrm{Al}_{2} \mathrm{O}_{3}$ la determina el contenido de fase líquida. Pero como quiera que no existen análisis completos para todos los ladrillos, en las figuras 3 y 4 se representan los límites de las temperaturas a aplicar en función del contenido de $\mathrm{Al}_{2} \mathrm{O}_{3}$, factor determinante del precio. En la figura 3 se encuentra en ordenadas la máxima temperatura de aplicación dada por el fabricante. La ordenada en la figura 4 es la temperatura $t_{a}$ del ensayo de resistencia a la compresión bajo carga a temperatura elevada. Esta es la temperatura a la que una probeta calentada bajo carga de $2 \mathrm{kp} / \mathrm{cm}^{2}$ se comprime $0,3 \mathrm{~mm}$ desde el punto de la máxima dilatación.

En ladrillos esta temperatura corresponde aproximadamente a la de aplicación. Tanto en las masas (fig. 3) como en los ladrillos (fig. 4) se ve que, con el contenido creciente de $\mathrm{Al}_{2} \mathrm{O}_{3}$, en la zona de menos de $50 \%$ la temperatura límite de aplicación o la temperatura, en el caso de los ladrillos, crece más rápidamente que en la zona de más del $50 \%$ de $\mathrm{Al}_{2} \mathrm{O}_{3}$. Esta relación se refiere únicamente a la carga térmica; pero con mayor con- 
tenido de alúmina cambian además otras propiedades de utilización, tales como la resistencia a la acción de las escorias, la resistencia al calor y otras.

T A B L A 1

Ladrillos empleados en el tubo rotatorio de hornos de vía seca cortos

\begin{tabular}{lll}
\hline Z o n a & \multicolumn{1}{c}{$\begin{array}{c}\text { Longitud de la zona } \\
\text { en diámetros D }\end{array}$} & \multicolumn{1}{c}{ Ladrillos empleados } \\
\hline Anillo de descarga & $0,5 \mathrm{D}$ & $\begin{array}{l}\text { Ladrillo con alto contenido de alúmina } \\
\text { Ladrillo rico en alumina } \\
\text { Ladrillo de chamota } \\
\text { Ladrillo de espinela }\end{array}$ \\
\hline Zona de enfriamiento & $4 \mathrm{D}$ & $\begin{array}{l}\text { Ladrillo con alto contenido de alúmina } \\
\text { Ladrillo de cromo-magnesita }\end{array}$ \\
\hline Zona de transición & $\begin{array}{l}\text { Ladrillo de dolomita } \\
\text { Ladrillo de cromo-magnesita }\end{array}$ \\
\hline Ladrillo de magnesita
\end{tabular}

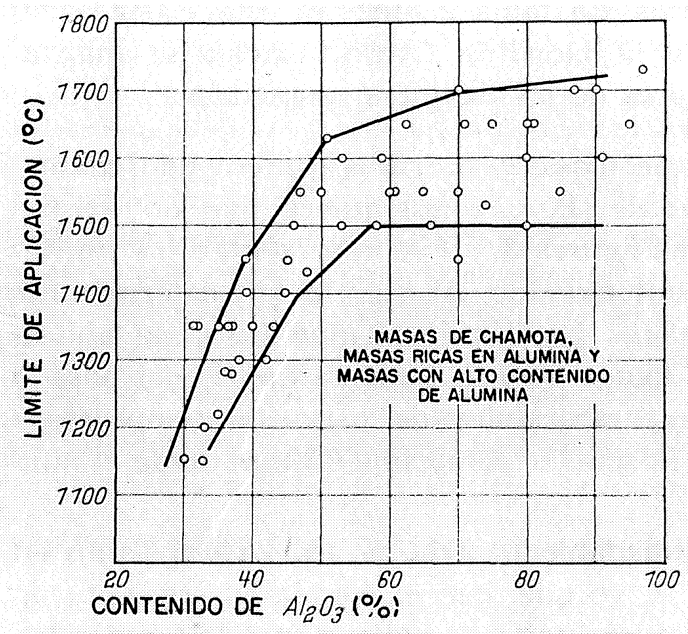

Fig. 3.-Temperaturas-límite de aplicación y contenido de $\mathrm{Al}_{2} \mathrm{O}_{3}$ de masas de chamota, masas ricas en alúmina y masas con alto contenido de alúmina.

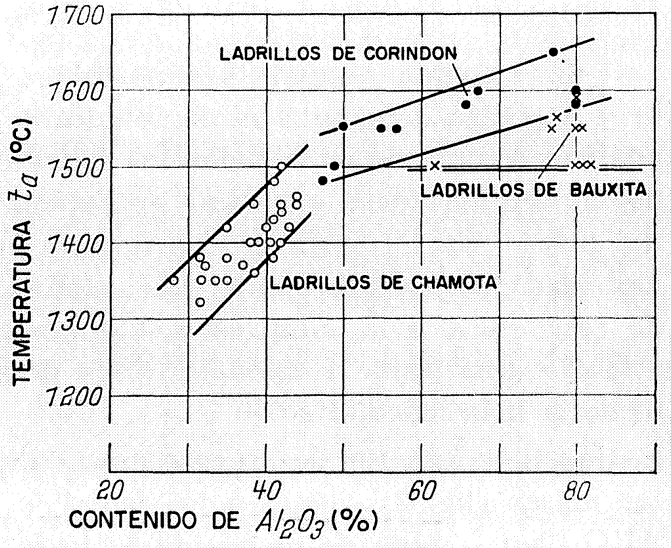

Fig. 4.-Temperatura y contenido de $\mathrm{Al}_{2} \mathrm{O}_{3}$ de ladrillos de chamota, ladrillos ricos en alúmina y ladrillos con alto contenido de alúmina. 


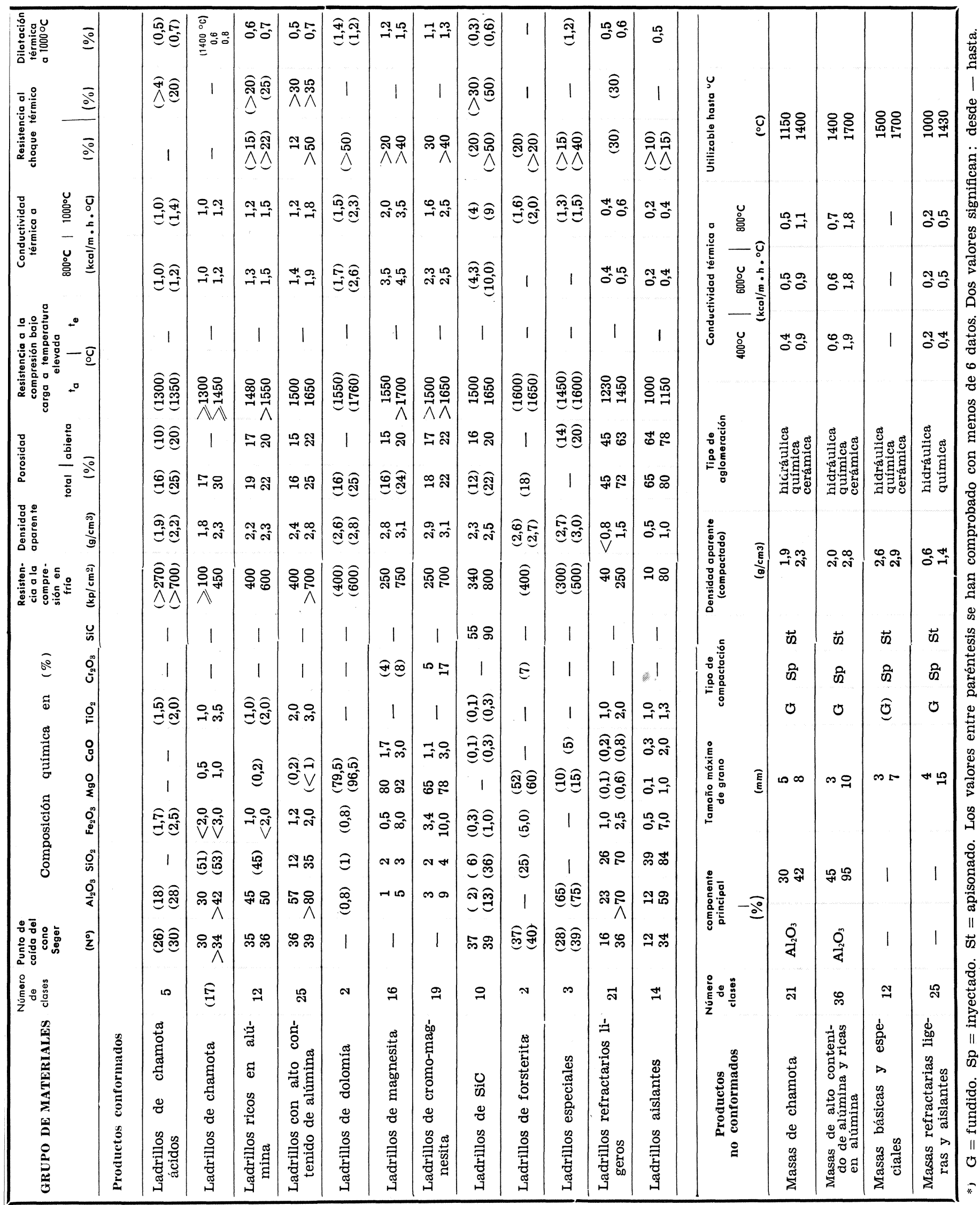


No se aprecia claramente una relación similar entre la temperatura $t_{a}$ y el contenido de $\mathrm{MgO}$, como componente principal entre ladrillos de magnesita y ladrillos de cromo-magnesita (fig. 5). Esto hay que atribuirlo a la menor validez de la temperatura $t_{a}$ para estos ladrillos.

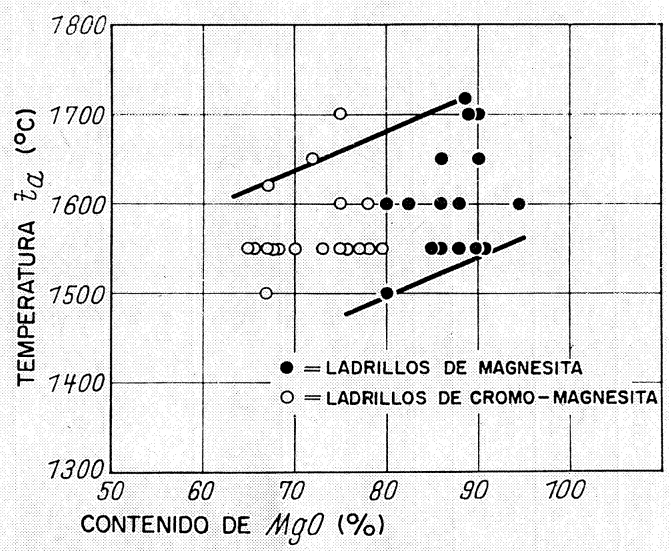

Fig. 5.-Temperatura $t_{a}$ y contenido de Mgo de ladrillos de magnesita y cromo-magnesita.

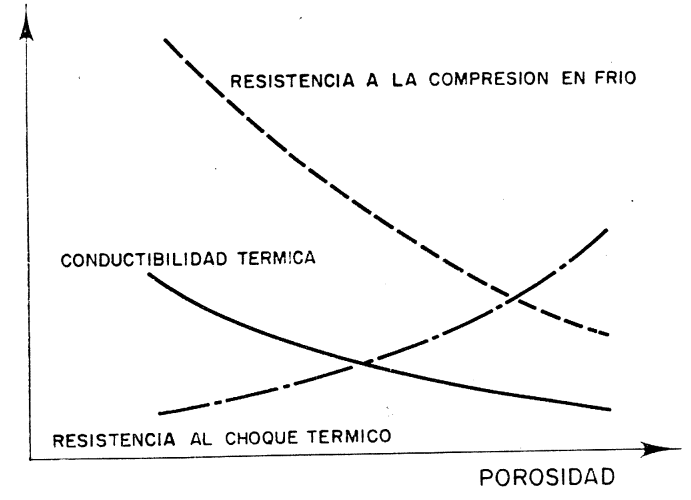

Fig. 6.-Influencia de la porosidad sobre resistencia a la compresión en frío, conductibilidad térmica y resistencia al choque térmico.

Los ladrillos refractarios son más o menos porosos. Los poros disminuyen la resistencia y la conductibilidad térmica, pero confieren a la sustancia sólida la posibilidad

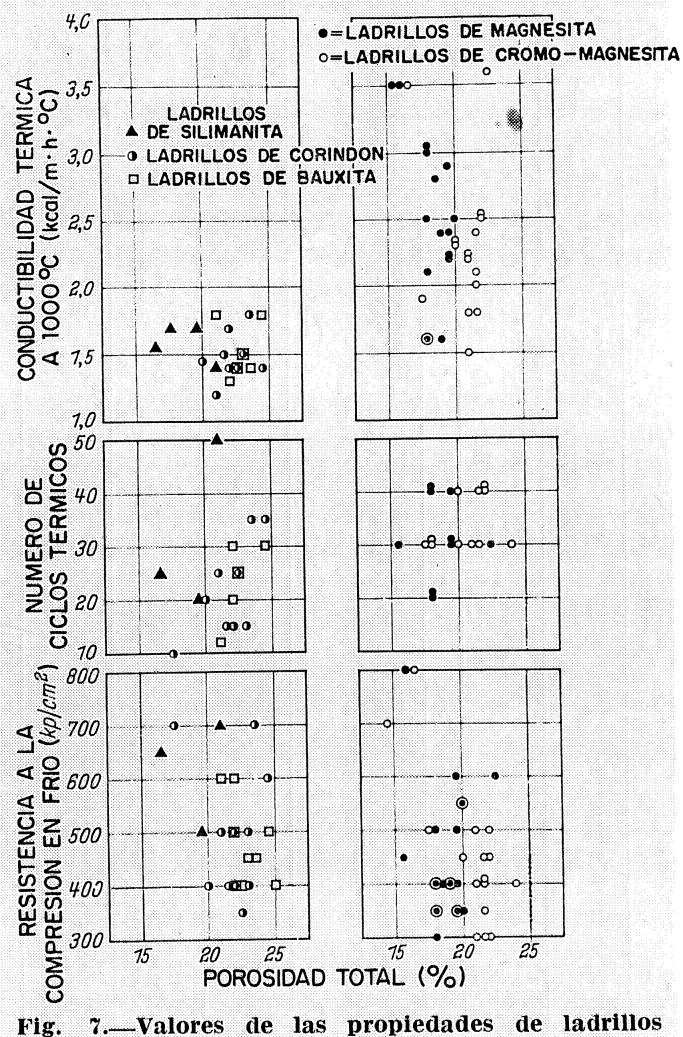

Fig. 7.-Valores de las pros de sinterización.
de zonas de de cambio de volumen en la mampostería al producirse cambios bruscos de temperatura. Por lo tanto, en ladrillos de igual composición, pero con contenido creciente de poros, es de esperar menor resistencia en frío, menor conductibilidad térmica y mejor comportamiento a los cambios bruscos de temperatura (fig. 6).

En la figura 7 se ha intentado demostrar estas relaciones en los valores sacados del catálogo de materiales refractarios para fábricas de cemento. Pero las relaciones esperadas casi no se perciben, lo que puede deberse al número relativamente bajo de valores y a la estrecha zona de dispersión de los índices de porosidad. También distintos métodos de comprobación de la conductibilidad térmica y del comportamiento a los cambios de temperatura pueden suministrar resultados diversos. La figura muestra que pequeñas diferencias significan poco en los valores comprobados que se mencionan de diversos resultados. 
Como quiera que los valores comprobados para diversos ladrillos —debidos también a una normalización aún no determinada - únicamente permiten una comparación imprecisa entre los diferentes productos y, por otro lado, como no es posible establecer una relación fija entre valores comprobados en el laboratorio y las exigencias en el lugar de utilización, al establecer un suministro de ladrillos debe ponerse más atención a la uniformidad que a la magnitud de los valores comprobados. La idoneidad de un tipo de ladrillo hay que establecerla, además, probándola realmente en el servicio del horno.

\section{AISLAMIENTO TERMICO}

Un revestimiento con buen poder calorífugo disminuye el consumo de calor en la cocción del clínker. En la zona de precalentamiento del tubo rotatorio se han generalizado los ladrillos refractarios ligeros como revestimiento de trabajo. Rara vez se emplean revestimientos de dos capas con la zona interior de chamota como revestimiento de trabajo y la cubierta exterior de ladrillo refractario ligero o ladrillo aislante para mejorar la protección. Los ladrillos de dos capas, que reúnen las dos cubiertas, han tenido poco exito.

La zona para ladrillos refractarios ligeros, en el horno rotatorio corto, termina en la zona de sinterización entre un tercio y la mitad de la longitud del horno. Ultimamente se han desarrollado de corindón granular, que poseen buen comportamiento hasta temperaturas de unos $1.600^{\circ} \mathrm{C}$ y pueden colocarse aún más cerca de la zona de sinterización. A temperaturas superiores a los $1.000^{\circ} \mathrm{C}$ se transmite, sin embargo, mucho más calor por radiación que por conducción, de tal forma que el aire ocluido ya no aiśla. La protección calorífuga de estos ladrillos no repercute, por lo tanto, en la parte exterior de la pared del horno hasta que las temperaturas no estén por debajo de los ya citados $1.000^{\circ} \mathrm{C}$.

El revestimiento refractario de la zona de transición se rellena frecuentemente con plaquitas de chamota de 40 a $60 \mathrm{~mm}$ de espesor. En cambio, cada vez más, se prescinde del relleno del revestimiento de la zona de sinterización, ya que en esta zona lo que mejor aisla es una costra suficientemente espesa. Solamente, en el caso de que el producto de la cocción no forma ningúna incrustación, se procederá a un relleno.

Una protección calorífuga perfeccionada de las paredes del precalentador ha sido realizada por el Dr. C. Otto de Bochum. Colocó el revestimiento de trabajo de ladrillos de chamota ácidos sobre planchas de materiales aislantes. Estas planchas se pueden colocar más fácil y rápidamente que los ladrillos aislantes mampuestos y acusan una mejor acción calorífuga.

\section{FORMATOS Y COLOCACION DE LOS LADRILLOS}

La construcción de grandes hornos rotatorios con diámetros de $5 \mathrm{~m}$ y más, ha llevado a discusiones sobre el más conveniente formato de ladrillos-dovela. Algunos fabricantes de ladrillos suministran, además de los formatos recomendados en la hoja informativa VDZ WE 9 (8), otros formatos con aproximadamente $75 \mathrm{~mm}$ de medida en el cono y para ladrillos básicos con la división $\pi / 3$ propuesta por Naredi (3), es decir, con una medida exterior constante de $103 \mathrm{~mm}$. La máxima conicidad de estos ladrillos garantiza una estabilidad mejor en el anillo. Si la prueba prolongada en el servicio no acusa defectos de calidad con respecto a ladrillos más pequeños, defectos que podrían producirse por contracción irregular al cocer los ladrillos, es de esperar que se introduzcan también para diámetros menores de hornos. 
El número total de formatos de ladrillos necesarios para hornos rotatorios de cemento podría reducirse entonces a 8 , ya que entonces podrían obtener, de igual forma, ladrillos de chamota y ladrillos básicos. El ladrillo $\pi / 3$ debiera quedar como única alternativa para los formatos recomendados en la hoja informativa VDZ WE 9, con el fin de no aumentar nuevamente el pequeño número de formatos, en ventaja de fabricantes y consumidores.

La industria del cemento ha exigido hace tiempo la caracterización (marcado) de la parte de los ladrillos que, una vez colocados, apunta hacia el recinto del horno, para, después del montaje, poder verificar su colocación correcta y el cumplimiento de las relaciones de mezcla en el anillo. La Dolomitwerk Wülfrath quiere marcar con una ranura o estría la cara del ladrillo que quedará orientada hacia el recinto del horno. Además, los formatos standard para hornos de 2, 4, $6 \mathrm{y}$, en el futuro, $8 \mathrm{~m}$ de diámetro debieran de marcarse de distinto modo para que, al colocarlos, no pueda haber confusiones.

En revestimientos de ladrillos se observa una tendencia a la colocación en seco. Hace 10 años se necesitaba aproximadamente $10 \%$ del peso de los ladrillos en mortero. Hoy en día se precisa únicamente de 5 a $8 \%$, porque aproximadamente un tercio de los ladrillos se coloca "a hueso" en el tubo rotatorio, es decir, sin mortero. Con esta forma de trabajar los ladrillos deben tener unas medidas muy precisas. Además, en el anillo hay que prever más juntas de dilatación que en la colocación con mortero. Como compromiso, Didier de Wiesbaden ha desarrollado un "papel de mortero" que por ambas caras tiene una capa fina de materiales de mortero y se introduce en las juntas.

Los ladrillos básicos se colocan desde hace tiempo también en chapas, con preferencia los ladrillos de dolomía y menos frecuentemente los ladrillos de magnesita y cromomagnesita. Las opiniones sobre el ahorro de tiempo en la colocación son muy diversas. Con tubos rotatorios totalmente redondos se colocan los ladrillos limpia, sencilla y rápidamente en chapas. Mayores deformaciones de la envolvente del horno, como pueden producirse por ejemplo debido a las rosetas de fuego, se pueden rectificar más fácilmente con mortero. Las chapas se sinterizan al llegar a altas temperaturas $\mathrm{y}$, por lo tanto, es más seguro el calentamiento con mampostería de mortero.

A fin de ahorrar a los consumidores la preparación del mortero, la Refratechnik de Göttingen suministra mortero preparado en bidones de $40 \mathrm{~kg}$, el cual puede tomarse directamente con la llana de los recipientes.

Para el dimensionado de las juntas de dilatación hay que observar los datos del fabricante correspondiente, ya que no sólo hay que tener en cuenta la dilatación térmica, sino también las posteriores contracciones y dilataciones de los ladrillos durante el servicio. Estas propiedades dependen de las materias primas, del prensado y de la cocción de los ladrillos. Corrientemente se introducen cartones en las juntas de dilatación, que al calentar se queman aun antes de que la mampostería haya alcanzado su dilatación total y, con ella, la unión sólida definitiva. Didier, de Wiesbaden, recomienda chapa de amianto que soporta temperaturas más elevadas.

\section{NUEVOS DESARROLLOS}

La Veitscher Magnesitwerke, de Viena, ha desarrollado el ladrillo - representado en la figura 8- que tiene medidas normales, pero con escotaduras en las superficies de apoyo para facilitar la adherencia de la costra. En la junta de los ladrillos se forman bolsas, que se rellenan del producto de la cocción y pueden anclar la costra de clínker en el revestimiento. 
La Plibrico, de Düsseldorf, ha montado planchas de $1 \mathrm{~m}$ de largo y $0,5 \mathrm{~m}$ de ancho (cada una corresponde a un paquete de unos 30 ladrillos), a modo de ensayo en un tubo rotatorio de 4,0 $\mathrm{m}$ de diámetro. Las planchas de hormigón de chamota se prefabricaron y se secaron a $400^{\circ} \mathrm{C}$. Se pueden llevar con una carretilla elevadora en el horno al lugar de colocación. Las planchas se fijaron con 2 tornillos, en sentido longitudinal, sobre la envolvente del horno. Los elementos han resistido hasta ahora 3 meses de servicio. Caso de obtener buenos resultados, se aumentarán las dimensiones de las planchas en el próximo ensayo. La colocación de las planchas la realizan dos montadores en vez de varios albañiles. Ya, en el primer ensayo, se logró el doble de rendimiento de colocación por hombre y hora, en comparación con el trabajo clásico de albañilería.

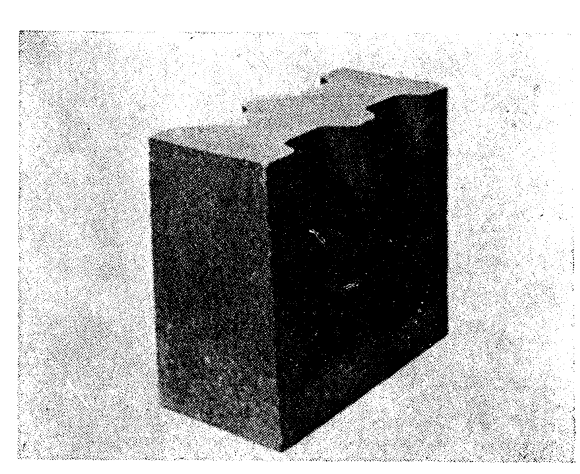

Fig. 8.-Ladrillo acuñado con escotaduras para mejor adherencia de la costra (Veitscher Magnesitwerke AG de Viena).

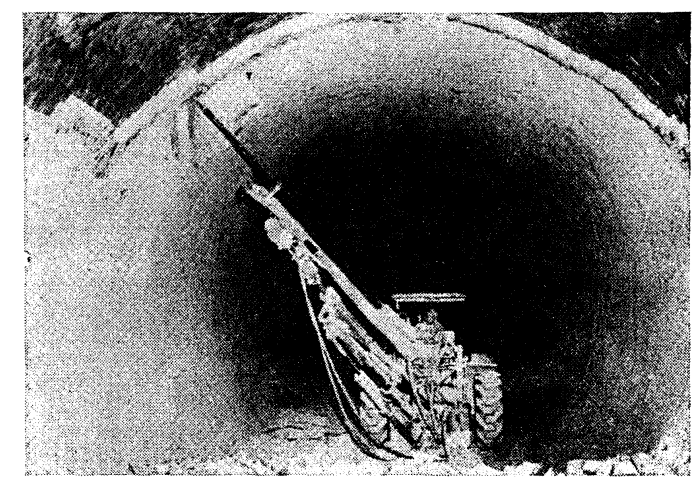

Fig. 9.-Aparato para romper el revestimiento refractario de horno rotatorio (según D. G. Shank).

Al mampostear los grandes hornos actuales, se transportan varios cientos de toneladas de ladrillos al tubo rotatorio. Una solicitud de patente de la Plibrico, de Düsseldorf, prevé el arriostramiento de los anillos en el horno ; los cantos interiores de dicho arriostramiento sirven de superficies de rodadura para los mecanismos de traslación, que además llevan una vía para carretillas elevadoras y aparatos similares. La vía puede quedar al girar el horno, lo cual es sobre todo ventajoso en trabajos de reparación si se gira con frecuencia.

Como quiera que romper la mampostería desgastada es muy costoso en grandes hornos, la firma Gierlichs, de Colonia, ha desarrollado un aparato con un martillo neumático elevable y giratorio sobre un mecanismo de traslación de oruga, para desprender el revestimiento. De USA se informa de un aparato similar montado sobre un cargador con neumáticos, tal como se representa en la figura 9. Este aparato pesa, con el dispositivo barrenador, 10 t. En 20-30 minutos puede cambiarse la barrenadora por una pala cargadora. El ahorro de tiempo al romper con este aparato es de $50 \%$ comparado con el trabajo manual; la gran seguridad de este método, en cuanto a la prevención de accidentes, es evidente.

\section{R E S U M E N}

Actualmente, los materiales refractarios se eligen, en primer lugar, en función de su durabilidad o de un comportamiento definido con precisión, con objeto de predecir una 
previsión a largo plazo de las reparaciones del revestimiento. El precio de venta más elevado de una calidad superior se amortiza fácilmente, pues el costo de las paradas de funcionamiento imprevistas, sobre todo en las instalaciones de gran tamaño, sobrepasará rápidamente el $2 \%$ comprendido en el precio de venta del cemento reservado a los revestimientos refractarios.

El empleo de morteros refractarios no se ha extendido en la industria cementera en la medida que era de prever. Esto se debe al hecho de que muchas fábricas de cemento no disponen de experiencia suficiente en materia de puesta en obra de las masas refractarias. En la Alemania Federal, la zona de cocción de los hornos rotativos se reviste actualmente con frecuencia de ladrillos de dolomía. Se han probado los ladrillos de carburo de silicio para el revestimiento de la descarga.

Los resultados a partir de ensayos de laboratorio son insuficientes para hacer previsiones del comportamiento en servicio real. La elección de los ladrillos a poner en fábrica depende, pues, de la experiencia. Por el contrario, la homogeneidad de las partidas puede controlarse gracias a los ensayos de laboratorio.

Los ladrilios refractarios ligeros aseguran una buena barrera térmica en la zona de precalentamiento ; en tanto que en la zona de cocción, esta misión se satisface por los depósitos sólidos (costra).

Se manifiesta una cierta tendencia a elegir la puesta en seco, en el caso de revestimiento con ladrillos, lo que exige juntas de dilatación cuidadosamente dimensionadas y ladrillos de gran estabilidad dimensional. La estera de amianto en lugar del amianto en planchas para las juntas de dilatación presenta una mayor seguridad durante la elevación de la temperatura del horno. Las operaciones de colocación y derribo del revestimiento puede facilitarse empleando máquinas rompedoras montadas sobre vehículo, así como carriles móviles para los carros elevadores.

\section{B I B L I O G R A F I A}

(1) Statistisches Jahrbuch für die Bundesrepublik Deutschland vom Statistischen Bundsemt, Wiesbaden, 1962-1969.

(2) RoutschkA, G.: Die Prüfung der- und Reparaturmassen im Spiegel der Literatur. Keramische Zeitschrift 20 (1968), 643 .

(3) NAREDI, R.: Neuere Verfahren der feuerfesten Auskleidung in der Zementindustrie. Zement-Kalk-Gips 22 (1969), 251.

(4) Struzik, E.: 5 Jahre feuerfeste Baumassen in deutschen Zementwerken. Zement-Kalk-Gips 22 (1969), 279.

(5) KÜNNECKE, M., und NAEFE, H.: Verbesserung der Futterhaltbarkeiten in Großdrehrohröfen durch großformatige Ziegelsteine und Hochbrandsteine. Zement-Kalk-Gins. 22 (1969), 271.

(6) Merkblatt WE 10 des Vereins Deutscher Zementwerke e. V., Düsseldorf: "Katalog feuerfester Stoffe für Zementwerke", 2. Auflage, November 1968, Selbstverlag.

(7) Konopicky, K.: Einteilung und Kennzeichnung dichter feuerfester Erzeugnisse einer Porigkeit unter $45 \%$. Stahl und Eisen 89 (1969), 238.

(8) Merkblatt WE 9 des Vereins Deutscher Zementwerke e. V., Düsseldorf : "Feuerfeste Steine für ZementDrehrohröfen". Mai 1966, Selbstverlag.

(9) Shank, D. G.: Dundee slashes cost with unique refractory peeler. Rock Products Vol. 72 (1969), H. $12,82$. 